\title{
Benefits of $\beta$ blockers in patients with heart failure and reduced ejection fraction: network meta-analysis
}

In this article by Saurav Chatterjee and colleagues (BMJ 2013;346:f55, doi:10.1136/bmj.f55), an error in the summary box was not spotted. The list of $\beta$ blockers in the second point

under "What is already known on this topic" should include

bisoprolol and not bucindolol. 